Article

\title{
Performance Analysis of Hard-Switching Based Hybrid FSO/RF System over Turbulence Channels
}

\author{
Hira Khalid ${ }^{1}$, Sajid Sheikh Muhammad ${ }^{1}$, Hector E. Nistazakis ${ }^{2, *}$ and George S. Tombras ${ }^{2}$ \\ 1 Department of Electrical Engineering, National University of Computer and Emerging Sciences, Lahore, \\ Islamabad 44000, Pakistan; khalid.hira28@gmail.com (H.K.); sm.sajid@nu.edu.pk (S.S.M.) \\ 2 Department of Electronics, Computers, Telecommunications and Control, Faculty of Physics, National and \\ Kapodistrian University of Athens, 15784 Athens, Greece; gtombras@phys.uoa.gr \\ * Correspondence: enistaz@phys.uoa.gr; Tel.: +30-210-7276710
}

Received: 30 April 2019; Accepted: 4 June 2019; Published: 6 June 2019

check for updates

\begin{abstract}
The hybrid system of free space optic (FSO) and radio frequency (RF) has come forth as alternative good solution for increasing demand for high data rates in wireless communication networks. In this paper, wireless networks with hard-switching between FSO and RF link are analyzed, assuming that at a certain time point either one of the two links are active, with FSO link having higher priority. As the signal-to-noise ratio (SNR) of FSO link falls below a certain selected threshold, the RF link is activated. In this work, it is assumed that the FSO link follows Gamma-Gamma fading due to the atmospheric turbulence effect whereas RF link experiences Rayleigh fading. To analyze the proposed hybrid model, analytical expressions are derived for the outage probability, bit error rate and ergodic capacity. A numerical comparison is also done between the performances of the proposed hybrid FSO/RF model and the single FSO model.
\end{abstract}

Keywords: Free Space Optics (FSO); Gamma-Gamma distribution; Rayleigh fading; ergodic capacity; bit error rate; Outage Probability

\section{Introduction}

Over the last two decades, we have seen the spread of wireless communication system over the entire globe. The number of wireless devices increases much rapidly than we have imagined. Radio-Frequency (RF) has always played a major role in our traditional wireless communication networks. The major problems of RF links are their low capacity and their high cost. RF is a costly source of communication because its frequency sub-bands are licensed. The exponential increase in the demand for high data rates and availability of frequency band for communication has pushed researchers to a limit where they must now explore other means of communication. The new wireless devices that are being added to our network demand for frequency band which is surmounting the capacity of the RF spectrum. It's the necessity of time that we start digging deeper into other frequency bands that are available in the electromagnetic spectrum.

Free-space optics has been proposed as a viable solution for higher data rates and emerged as a competitor for traditional communication systems. FSO operates over an unregulated band with the ability to provide services up to a few kilometers. FSO has astonished us with its capabilities and the speed it offers. Recently in 2018, German aerospace center, i.e., DLR, in collaboration with ADVA optical networking has set a record of 13.16 terabits/sec operational over $10.45 \mathrm{~km} \mathrm{[1].} \mathrm{Although} \mathrm{FSO}$ system can achieve very high data rates at a low cost, it has its own demerits as well. Thus, the FSO cannot completely replace the RF system everywhere. The impact of the atmosphere is not the same on FSO and RF. Attenuation is mainly caused by scattering and absorption. Clouds, snow and rain are the causes for scattering (also known as aerosol scattering), whereas, absorption of FSO signal is 
mainly due to carbon dioxide and water particles. The reason behind significant attenuation of FSO link due to scattering is the size of fog particle, which is comparable to the operating wavelength of the optical signal. This kind of scattering is called Mie scattering and are generally applicable to horizontal links close to the earth's surface. FSO signal is not sensitive to rain but degrades significantly in fog. For RF signal, frequencies below $10 \mathrm{GHz}$ are merely affected by the fog droplets, whereas, for frequencies above $10 \mathrm{GHz}$ faces significant scattering [2]. RF signal is not very sensitive to the fog, but its performance deteriorates in rain. So, FSO and RF can be good complimentary systems to be deployed together.

For the last few years, researchers have been working on hybrid systems with FSO to enhance its performance and capabilities. In [3], hybrid power line communication (PLC) and visible light communication (VLC), also known as FSO, was studied by practical demonstration. This hybrid system is analyzed based on the maximum range, the connectivity and speed. In [4], possibilities of hybrid FSO/RF systems were investigated. Concept of receiver diversity is proposed in which the receiver takes the copies of the data and combines it to recover the originally transmitted signal. These copies are taken from parallel working FSO and RF link simultaneously. In [2], the effects of different weather conditions for hybrid FSO/RF link were studied. A setup for hybrid FSO/RF system was installed to experimentally measure the real-time measurements of attenuation. Comparative study for attenuation was evaluated by a model by Kim, Kruse and Naboulsi. In [5], the authors analyzed the availability of FSO-only system and hybrid FSO/RF system. They practically showed that FSO-only system can achieve $99.999 \%$, i.e., five nines, availability for a distance of $140 \mathrm{~m}$ only, whereas, a hybrid FSO/RF system could increase the availability distance for five nines. In [6], hybrid FSO/RF was tested in a practical environment using adaptive combining. The receiver retrieved the original data out of two links by using a maximal ratio combining (MRC) technique. Another relay scheme was proposed in $[7,8]$, where the relay system was analyzed for two nodes with the dual hop between FSO and RF link. In [9], link adaptation technique was used for a hybrid system where RF-FSO dual link activated as the threshold of FSO link went below a certain threshold. Dual FSO threshold scheme was also discussed which is effective to avoid frequent switching when the weather conditions change rapidly. In [10], performance was evaluated using the selective combining (SC) technique for the hybrid system. Authors in [11] have proposed a rate less coding scheme using off-the-shelf Raptor code in hybrid FSO/RF system. They have compared the hybrid system with and without rate less coding to prove that hybrid system with rate less coding can fully utilize the available RF and FSO link resources.

In this paper, a hybrid FSO/RF system is proposed to increase both the reliability and availability of the network. This system is analyzed assuming that FSO follows Gamma-Gamma fading whereas RF link follows Rayleigh fading. Intensity modulation with direct detection (IM/DD) using on-off keying $(\mathrm{OOK})$ is implemented. The system under consideration is analyzed on the bases of outage probability (OP), bit error rate (BER) and channel's (average) ergodic capacity. To the best of our knowledge, the performance of the hybrid system with Gamma-Gamma fading for FSO and Rayleigh fading for RF has never been investigated before, by means of its OP, BER and ergodic capacity estimation.

It is considered that information is transmitted with hard-switching either FSO or RF link depending on the circumstances, i.e., only one of the links will operate at each specific time. For the time at which the SNR of FSO link remains above a certain selected threshold, the system keeps working on FSO. However, when the quality of FSO link degrades to a level below the selected threshold, the system moves to RF link. This threshold for FSO link is given by $\gamma_{t h}{ }^{F S}$. There is another case when the system can completely go into outage and it's when the RF signal loses its SNR and goes below the specific threshold $\gamma_{t h}{ }^{R F}$. For simplicity, it is assumed that at least one link remains active at a certain time and the system does not interrupt the total Hybrid FSO/RF link. As one link remains active at a time, the transmitter will have to consume less power and receiver design would be simpler as it will not require any combination techniques to extract data like in $[9,10]$. Figure 1 portrays the basic idea of the hybrid system while Figure 2 shows the state transition diagram for this hard-switching between 
FSO and RF link of the Hybrid FSO/RF system under consideration. Weather condition can change rapidly and cause frequent transitions between FSO and RF link. It is assumed that the system does not experience abrupt changes in turbulence and the weather conditions are steady.

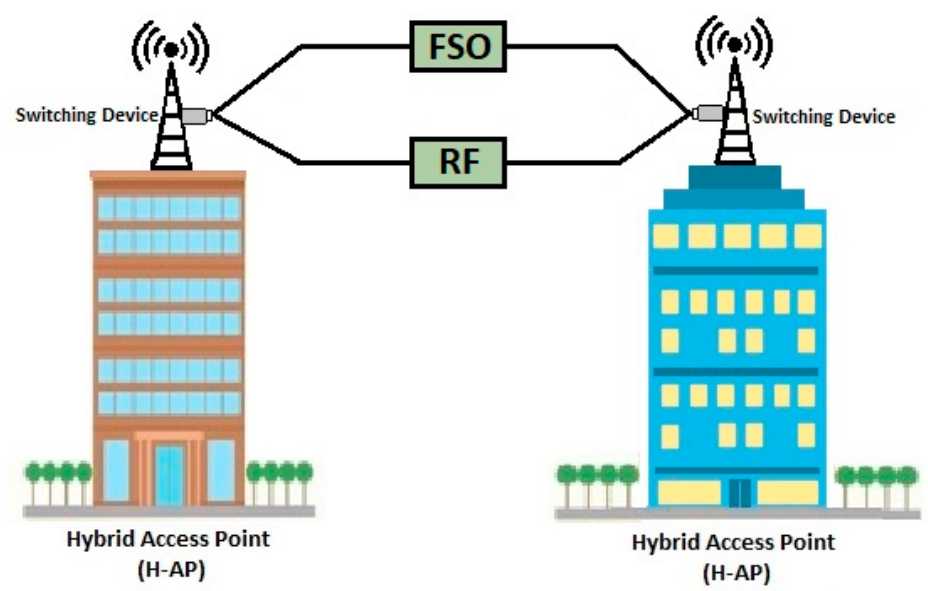

Figure 1. System block diagram.

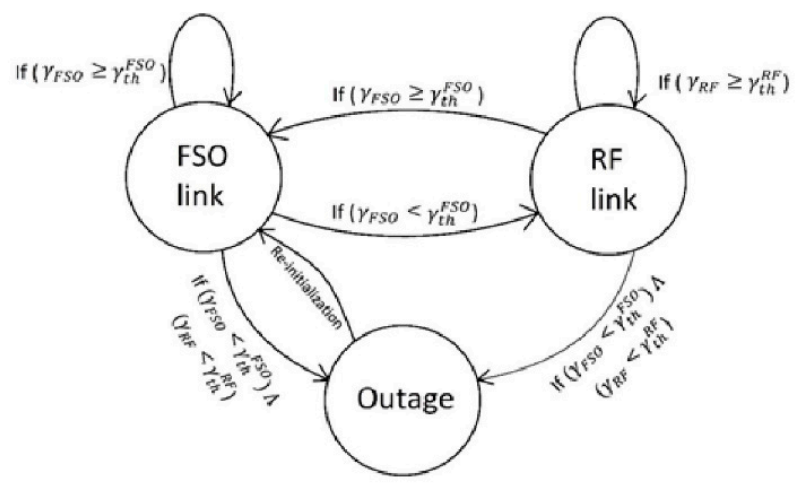

Figure 2. Transition diagram for hard-switching.

The remainder of this work is organized as follows: Section 2 will provide a brief introduction of the system model and the derivation of performance analyzing parameters will be achieved in subsection A, B and C, respectively. Section 3 will present the numerical analyzation. Finally, Section 4 will conclude this paper.

\section{System Model}

Considering a hybrid FSO/RF system in which it is assumed that FSO link is having Gamma-Gamma fading, whereas, RF link follows Rayleigh fading. FSO utilize IM/DD with OOK scheme. For simple receiver implementation, it is assumed that only one of the links is active at a certain point. A simple mathematical expression that presents the channel from FSO transmitter to the photo-detector is given as $[12,13]$ :

$$
y=\eta P_{o} x+w
$$

where $\eta$ is the efficiency of a photodetector, which for simplicity is assumed to be equal to one, $x$ represents the transmitted FSO symbols, $P_{o}$ is the optical power and $w$ is considered Gaussian distributed random variable with unitary variance and zero mean. For detection, low and stationary aerosol scattering or light haze is assumed with transmission efficiency $\eta$ comparable to unity. The signal-to-noise ratio at FSO receiver can be expressed as [6]: 


$$
\gamma_{F S O}=\frac{E_{s} \mu^{2} R^{2} P_{F S O}^{2} G_{F S O}^{2}}{\sigma_{F S O}^{2}} I^{2},
$$

where $E_{s}$ is the average symbol energy, $\mu$ is the modulation index, $R$ is the photodetector's responsivity, $P_{F S O}$ is the optical power transmitted, $\sigma_{F S O}$ represents the shot noise variance at photodetector which can be modeled as additive white Gaussian noise (AWGN), I stands for the received optical irradiance and $G_{F S O}$ is the attenuation of optical power which can be estimated by the Beers-Lambert law.

Point-to-Point RF link operating at $58 \mathrm{GHz}$ is assumed as the backup link for the above introduced FSO channel. The specific RF frequency band is comparatively less sensitive to deep fades due to the rain [2]. For short links-range, i.e., shorter than $2 \mathrm{~km}$, the overall performance of the hybrid system practically depends only on the FSO channel and not on the supporting RF channel.

Using Friis transmission equation, the channel power gain is given as [12]:

$$
G_{R F}=G_{t x} G_{d} G_{r x}
$$

where, $G_{t x}$ and $G_{r x}$ are the transmitter's and receiver's antenna gains, respectively and $G_{d}$ is the directive gain of the isotropic antenna.

\subsection{Outage Probability}

It is assumed that the FSO link follows Gamma-Gamma fading distribution so the corresponding probability density function (PDF) for the SNR is given as [14,15]:

$$
f_{F S O}=\frac{2(\alpha \beta)^{(\alpha+\beta) / 2} I^{(\alpha+\beta-2) / 2}}{\Gamma(\alpha) \Gamma(\beta)} K_{\alpha-\beta}(2 \sqrt{\alpha \beta I}),
$$

where $\Gamma($.$) is the gamma function, K_{v}($.$) is the modified Bessel function of second kind and order v$, while $\alpha, \beta$ are the parameters of the distribution and can be estimated directly from the link's parameters and the atmospheric turbulence strength [15]. For FSO applications the specific parameters are given as [16]:

$$
\alpha \cong \exp \left[\frac{0.49 \sigma_{R}^{2}}{\left(1+1.11 \sigma_{R}^{12 / 5}\right)^{7 / 6}}\right]-1
$$

and

$$
\beta \cong \exp \left[\frac{0.51 \sigma_{R}^{2}}{\left(1+0.69 \sigma_{R}^{12 / 5}\right)^{7 / 6}}\right]-1
$$

These parameters are related to Rytov variance $\sigma_{R}^{2}$ and here, the plane wave propagation without the inner scale is assumed. Thus, the Rytov variance is given as, [12]:

$$
\sigma_{R}^{2}=1.23\left(\frac{2 \pi}{\lambda}\right)^{7 / 6} C_{n}^{2} v^{11 / 6}
$$

where $\lambda$ is the operational wavelength of the FSO link, $v$ is the link's length and $C_{n}^{2}$ is the refractive-index structure parameter which actually represents the atmospheric turbulence strength. Generally, fluctuations in refractive index for wave propagation through turbulence atmosphere are considered isotropic in nature. But near the earth's surface, i.e., $2-4 \mathrm{~m}$ above the surface, the propagation is anisotropic due to moving atmosphere and stationary ground interaction [17]. Mutual coherence functions (MCF) for one ccc-dimensional vertical and horizontal directions are measurably different in anisotropic turbulence regimes. For our proposed model, it is assumed that communication established at the rooftop is present in a zone where the turbulence can be taken into account as is otropic and much lower than near ground turbulence. For these cases, the value of $C_{n}^{2}$ could be around $10^{-17} \mathrm{~m}^{-2 / 3}$, [18]. 
Next, the PDF of $\gamma_{F S O}$ can be estimated from (4) using a power transformation of random variables as $[6,13]$ :

$$
f_{F S O}\left(\gamma_{F S O}\right)=\frac{\left(\alpha \beta / \sqrt{\bar{\gamma}_{F S O}}\right)^{\frac{(\alpha+\beta)}{2}} \gamma_{F S O}{ }^{\frac{\alpha+\beta-4}{4}}}{\Gamma(\alpha) \Gamma(\beta)} K_{\alpha-\beta}\left(2 \sqrt{\alpha \beta \sqrt{\frac{\gamma_{F S O}}{\bar{\gamma}_{F S O}}}}\right)
$$

where $\gamma_{F S O}$ and $\bar{\gamma}_{F S O}=\frac{E_{S} \mu^{2} R^{2} P_{F S O}^{2} G_{F S O}^{2}}{\sigma_{F S O}^{2}} E[I]^{2}$ stand for the instantaneous and the expected electrical SNR, respectively, while the symbol $E[$.$] stands for the expected value and here, taking the value$ $E[I]=1,[13]$.

On the other hand, the RF link experiences Rayleigh fading as it is subjected to a multi-path effect. Accordingly, the signal to noise ratio corresponds to following exponential PDF [9]:

$$
f_{R F}\left(\gamma_{R F}\right)=\frac{1}{\overline{\gamma_{R F}}} e^{-\gamma_{\mathrm{RF}} / \overline{\gamma_{R F}}}
$$

where $\overline{\gamma_{R F}}$ is the time expected SNR of the received signal just before the modulated envelope is detected. To compute outage probability for a fixed transmission rate $R_{0}(\mathrm{bps} / \mathrm{Hz})=\log _{2}\left(1+\gamma_{\text {th }}\right)$ where, $\gamma_{t h}=2^{R_{0}}-1$ is the receiver's SNR threshold. The probability for which end-to-end SNR of the system is less than specific threshold $\gamma_{t h}$ is called outage probability, [6,19].

Thus, the outage probability either for the FSO link or the alternative RF connection estimated as [20]:

$$
\mathrm{P}_{\text {outage }}=\operatorname{Pr}\left(\mathrm{SNR}<\gamma_{\text {th- }}\right),
$$

where the subscript th- $x$, represents either th-FSO or th-RF depending on the SNR threshold value of each specific communication link, i.e., FSO or RF link, respectively.

The outage probability can be estimated using the cumulative distribution function (CDF) of the FSO link SNR. The total outage probability is given as [19]:

$$
F_{\text {out }}\left(\gamma_{\text {th-FSO }}, \gamma_{\text {th-RF }}\right)=F^{F S O}\left(\gamma_{\text {th-FSO }}\right) F^{R F}\left(\gamma_{\text {th-RF }}\right)
$$

where the CDF for the Gamma-Gamma FSO link is given as, [20]:

$$
F^{F S O}\left(\gamma_{t h-F S O}\right)=\frac{(a b)^{\frac{a+b}{2}}}{\Gamma(a) \Gamma(b)}\left(\frac{\gamma_{t h-F S O}}{\bar{\gamma}_{F S O}}\right)^{\frac{a+b}{4}} G_{1,3}^{2,1}\left(a b \sqrt{\frac{\gamma_{t h-F S O}}{\bar{\gamma}_{F S O}}} \mid \begin{array}{c}
1-\frac{a+b}{2} \\
\frac{a-b}{2}, \frac{b-a}{2},-\frac{a+b}{2}
\end{array}\right)
$$

where $G_{p, q}^{m, n}[\cdot]$ stands for the Meijer G-function, [21].

Furthermore, the CDF for the RF link is obtained by integrating (9), [22]:

$$
\mathrm{F}^{\mathrm{RF}}\left(\gamma_{\mathrm{th}-\mathrm{RF}}\right)=\int_{0}^{\gamma_{\text {th }}} f_{R F}\left(\gamma_{R F}\right)=1-e^{-\gamma_{\text {th }-R F} / \overline{\gamma_{R F}}}
$$

By substituting (12) and (13) into (11) we obtain:

$$
F_{\text {out }}\left(\gamma_{\text {th-FSO }}, \gamma_{\text {th-RF }}\right)=\frac{(a b)^{\frac{a+b}{2}}}{\Gamma(a) \Gamma(b)}\left(\frac{\gamma_{t h-F S O}}{\bar{\gamma}_{F S O}}\right)^{\frac{a+b}{4}} G_{1,3}^{2,1}\left(a b \sqrt{\frac{\gamma_{t h-F S O}}{\bar{\gamma}_{F S O}}} \mid \begin{array}{c}
1-\frac{a+b}{2} \\
\frac{a-b}{2}, \frac{b-a}{2},-\frac{a+b}{2}
\end{array}\right)\left(1-e^{-\gamma_{\text {th }}-R F} / \overline{\gamma_{R F}}\right)
$$

The above equation is a closed form expression for the outage probability of the hybrid FSO/RF system which is governed by the factors of gamma-gamma and Rayleigh distribution, respectively.

\subsection{Bit Error Rate}

For average BER calculations, it is assumed that the signal is modulated with OOK scheme and is then transmitted on either of the active FSO or RF link. It is further assumed that both the links operate 
at the data rate $R_{0}$. The conditional error probability for RZ-OOK, as a function of instantaneous SNR $\gamma$ is given by [23]:

$$
P(e \mid \gamma)=B E R_{R Z-O O K}=\frac{1}{2} \operatorname{erfc}\left(\frac{1}{2} \sqrt{\gamma}\right),
$$

The average BER for hybrid FSO/RF system, during a non-outage period, can be written as, [19]:

$$
B E R_{F S O / R F}=\frac{B_{F S O}\left(\gamma_{\text {th-FSO }}\right)+F^{F S O}\left(\gamma_{\text {th-FSO }}\right) \times B_{R F}\left(\gamma_{\text {th-RF }}\right)}{1-F_{\text {out }}}
$$

where $B_{F S O}($.$) is average BER for the time where the FSO link is activated and B_{R F}($.$) presents average$ BER for the time where RF link is activated. $F^{F S O}\left(\gamma_{t h-F S O}\right)$ and $F_{o u t}$ are given from (14) and (18), respectively. These terms can be defined as [19]:

$$
B_{F S O}\left(\gamma_{t h-F S O}\right)=\int_{\gamma_{\text {th }}}^{\infty} P\left(e \mid \gamma_{F S O}\right) f_{F S O}\left(\gamma_{F S O}\right) d\left(\gamma_{F S O}\right)
$$

and

$$
B_{R F}\left(\gamma_{t h-R F}\right)=\int_{\gamma_{\text {th }}}^{\infty} P\left(e \mid \gamma_{\mathrm{RF}}\right) f_{R F}\left(\gamma_{\mathrm{RF}}\right) d\left(\gamma_{\mathrm{RF}}\right)
$$

By substituting (8) and (15) into (17), the following integral for the estimation of the average BER for the FSO link is obtained:

$$
B_{F S O}\left(\gamma_{t h-F S O}\right)=\int_{\gamma_{t h-F S O}}^{\infty} \frac{K_{\alpha-\beta}\left(\alpha \beta / \sqrt{\gamma_{F S O}}\right)^{\frac{\alpha+\beta+1}{2}} \operatorname{erfc}\left(\frac{\sqrt{\gamma_{F S O}}}{2}\right) \gamma_{F S O} \frac{\alpha+\beta-3}{4}}{\Gamma(\alpha) \Gamma(\beta)} d\left(\gamma_{F S O}\right)
$$

While by substituting (9) and (15) into (18) the corresponding integral for the average BER of the RF link, is given as:

$$
B_{R F}\left(\gamma_{t h-R F}\right)=\int_{\gamma_{t h-R F}}^{\infty} \frac{\operatorname{erfc}\left(\frac{\sqrt{\gamma_{\mathrm{RF}}}}{2}\right) e^{-\gamma_{\mathrm{RF}} / \overline{\gamma_{\mathrm{RF}}}}}{2 \times \overline{\gamma_{\mathrm{RF}}}} d\left(\gamma_{\mathrm{RF}}\right),
$$

Then by substituting (12), (19) and (20) into (16) the average BER of the whole hybrid FSO/RF system can be estimated.

\subsection{Ergodic Capacity}

The capacity of the hybrid FSO/RF channel under consideration is estimated as, [19]:

$$
C=C_{F S O}\left(\gamma_{t h-F S O}\right)+F^{F S O}\left(\gamma_{t h-F S O}\right) C_{R F}\left(\gamma_{t h-R F}\right),
$$

where, $C_{F S O}($.$) or C_{R F}($.$) is the capacity of the system when only FSO or RF link is active, respectively$ and is given as, [19]:

$$
C_{\Xi}\left(\gamma_{t h-\Xi}\right)=\int_{\gamma_{t h-\Xi}}^{\infty} W_{\Xi} \log _{2}\left(1+\gamma_{\Xi}\right) f_{\Xi}\left(\gamma_{\Xi}\right) d\left(\gamma_{\Xi}\right)
$$

where the subscript $\Xi$ stands either for the FSO or RF link, while $W_{F S O}$ and $W_{R F}$ represent the corresponding bandwidths. Thus, by substituting (8) into (22) we conclude to the following integral for the estimation of average capacity for the FSO link, [12]:

$$
C_{F S O}\left(\gamma_{t h-F S O}\right)=\int_{\gamma_{t h-F S O}}^{\infty} W_{F S O} \log _{2}\left(1+\gamma_{F S O}\right) \frac{\left(\alpha \beta / \sqrt{\bar{\gamma}_{F S O}}\right)^{\frac{(\alpha+\beta)}{2}} \gamma_{F S O}\left(\frac{\alpha+\beta}{4}\right)-1}{\Gamma(\alpha) \Gamma(\beta)} K_{\alpha-\beta}\left(2 \sqrt{\frac{\alpha \beta \gamma_{F S O} 1 / 2}{\sqrt{\bar{\gamma}_{F S O}}}}\right) d\left(\gamma_{F S O}\right)
$$


and the corresponding expression for the average capacity for the RF link, through Equation (22), is given as:

$$
C_{R F}\left(\gamma_{\text {th-RF }}\right)=\int_{\gamma_{\text {th }-R F}}^{\infty} W_{R F} \log _{2}\left(1+\gamma_{R F}\right) \frac{1}{\overline{\gamma_{R F}}} e^{-\gamma_{\mathrm{RF}}} / \overline{\overline{\gamma_{R F}}} d\left(\gamma_{R F}\right)
$$

By integrating by parts, the integral of Equation (24) can be solved and we conclude to the following closed-form mathematical expression for the average capacity of the RF link under consideration:

$$
C_{R F}\left(\gamma_{t h-R F}\right)=\frac{W_{R F}}{\ln (2)} e^{-\frac{\gamma_{t h-R F}}{\bar{\gamma}_{R F}}}\left[E_{i}\left(\frac{\gamma_{t h-R F}+1}{\overline{\gamma_{R F}}}\right) e^{\frac{\gamma_{t h-R F}+1}{\bar{\gamma}_{R F}}}+\ln \left(\gamma_{t h-R F}+1\right)\right]
$$

where $E_{i}($.$) is the exponential integral function.$

By substituting (23) and (25) into (21), the average capacity of the whole hybrid FSO/RF system under consideration can be estimated.

\section{Numerical Results}

In this section, numerical examples are presented to illustrate our analysis. It is assumed that the transmitted power is equal to $320 \mathrm{~mW}$ for the FSO link and 16mW for RF. Optical receiver responsivity $R$ is fixed at a typical value of $0.5 \mathrm{~A} / \mathrm{W}$ and the variance of shot noise for the FSO is $\sigma_{F S O}=10^{-14} \mathrm{~A}^{2}$. For Gamma-Gamma distribution, typical values for strong atmospheric turbulence are selected to be $\alpha=2.064, \beta=1.342$, [6], while the typical value of refractive-index structure parameter is used for light haze, i.e., $C_{n}^{2}=1.8 \times 10^{-14} \mathrm{~m}^{-2 / 3}$. This value of $C_{n}^{2}$ is considerable for visibility range of $4-10 \mathrm{~km}$, so with our selected parameters, the distance between the transmitter and the receiver must not be more than $10 \mathrm{~km}$, [12]. The threshold for both links of the hybrid communication system is fixed at $\gamma_{t h}=5 \mathrm{~dB}$. Additionally, it is assumed that RF link is always available and so the system never goes into complete outage. The antenna gains of transmitter $G_{t x}$ and receiver $G_{r x}$ are assumed to be $16 \mathrm{dBi}$ and $30.4 \mathrm{dBi}$, respectively, which are typical values for such type of equipment.

In Figure 3, using the obtained Equation (14), the OP is presented for three different cases as a function of the average electrical SNR for the FSO link at the receiver. The red curve shows the OP for FSO-only case which means that here RF link remains inactive for all the values of average SNR of FSO i.e., even when average SNR of FSO link goes below the selected threshold for switching. The pink and blue curves are for hybrid FSO/RF system for which average SNR of RF link is kept constant at $5 \mathrm{~dB}$ and $10 \mathrm{~dB}$, respectively [19]. It can be seen from Figure 3 that the reliability of hybrid FSO/RF system surmounts the other case. We can also observe that for a hybrid system, the reliability improves by increasing the average SNR for the RF, i.e., performance is better for larger SNR values for the RF link.

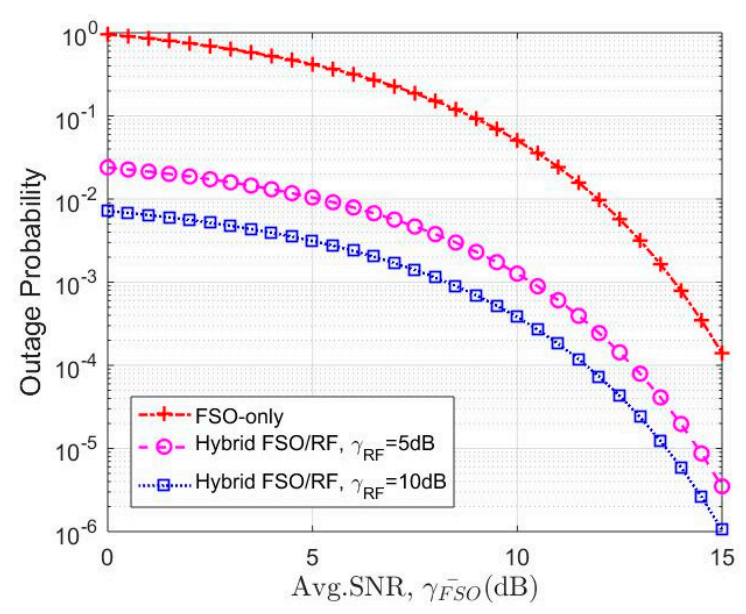

Figure 3. Probability as a function of average SNR of FSO link. 
In Figure 4, using the Equations (16), (19) and (20), shows the variations in average BER versus $\bar{\gamma}_{F S O}$. It can be observed that by keeping the threshold fixed and with high quality of RF link, hybrid FSO/RF system shows improvement in performance as the value of $\bar{\gamma}_{F S O}$ increases. For analyses, average SNR threshold is kept at $5 \mathrm{~dB}$ for FSO link. Improvement in BER can be seen because, in lieu of switching the transmission off, a feeble link is used for transmission and to keep the communication channel to be always available. With a better quality of radio frequency link, significant melioration in performance can be seen with varying SNR of FSO link. This effect can be observed from pink and blue curves of the graph in Figure 4 that high SNR link, i.e., $\gamma_{R F}=10 \mathrm{~dB}$, [19], has less outage probability than low SNR link, i.e., $\gamma_{R F}=5 \mathrm{~dB}$, [19]. Better quality of the backup link, i.e., RF, improves the overall performance of the hybrid system, as expected.

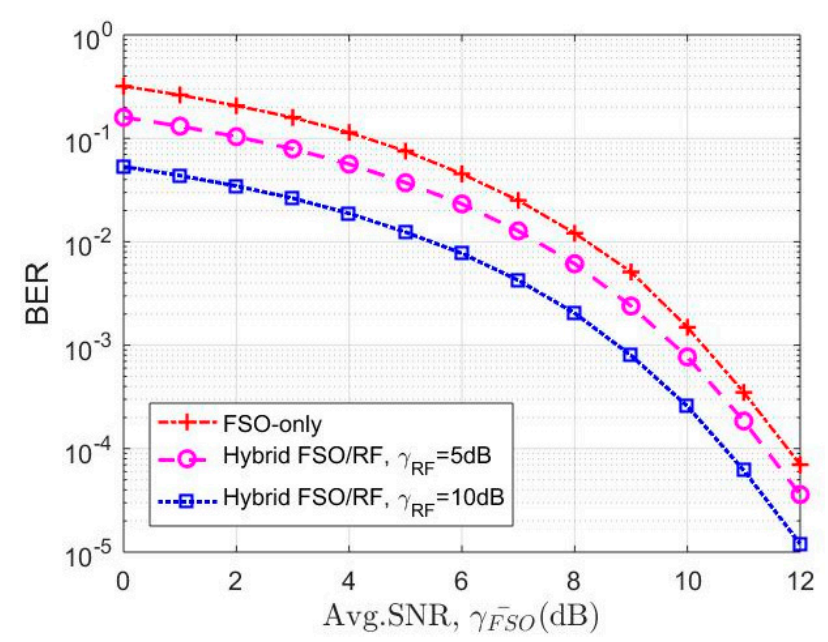

Figure 4. Bit error rate as a function of average SNR of FSO link.

The three curves in Figure 5, using Equations (21), (23) and (25), shows capacities of FSO-only, hybrid system with $\gamma_{R F}=5 \mathrm{~dB}$ and hybrid system with $\gamma_{R F}=10 \mathrm{~dB}$, [19]. The result is as expected, the hybrid FSO/RF system shows improvement in the ergodic capacity of the system especially at low average SNR of FSO link $\bar{\gamma}_{F S O}$. Over low $\bar{\gamma}_{F S O}$ the capacity of only FSO link is far less than that of hybrid FSO/RF, but at higher $\bar{\gamma}_{F S O}$ its capacity improves also. At higher $\bar{\gamma}_{F S O}$ the system operates more on FSO link instead ofactivating available RF link. For this reason, the capacity shown by a red curve for FSO-only approaches the curve of hybrid FSO/RF system as the two cases converge. It should be mentioned here that for marginal cases of our work, our obtained results appearing in Figures 3-5, can be compared with previous studies [12,19]. From such comparisons, it is evident that there is a good agreement in results. 


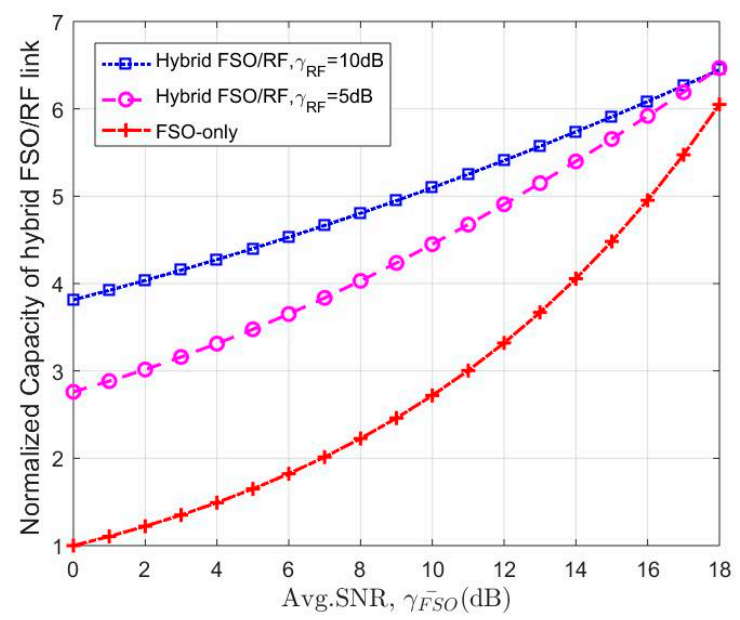

Figure 5. Normalized capacity C/W (bits/sec/Hz) versus average SNR of FSO link.

\section{Conclusions}

In this work, performance availability and reliability of hybrid FSO/RF system based on hard-switching are analyzed. The analysis is done based on three significant metrics for the communication systems known as outage probability, BER and ergodic capacity. The performance of the proposed hybrid FSO/RF model shows significant improvement and better performance in terms of availability and reliability as compared to conventional single FSO model. RF link with higher SNR further stables the hybrid FSO/RF system and gives much better results for the whole link.

Author Contributions: Conceptualization, H.K. and S.S.M.; methodology, H.K. and S.S.M.; software, H.K.; validation, H.K., S.S.M. and H.E.N.; formal analysis, H.K. and S.S.M.; investigation, H.K., S.S.M., H.E.N. and G.S.T.; resources, H.K., S.S.M., H.E.N. and G.S.T.; writing-original draft preparation, H.K. and S.S.M.; writing一review and editing, H.K., S.S.M. and H.E.N.; supervision, S.S.M.; funding acquisition, H.E.N., G.S.T.

Funding: This work has received funding from the European Union's Horizon 2020 research and innovation program under grant agreement No: 777596.

Conflicts of Interest: The authors declare no conflict of interest.

\section{References}

1. Schutz, A. DLR and ADVA Set a New World Record in Optical Free-Space Data Transmission. Available online: https://www.dlr.de/dlr/en/desktopdefault.aspx/tabid10989/1769_read-27323/\#/gallery/30516 (accessed on 15 April 2018).

2. Nadeem, F.; Kvicera, V.; Awan, M.; Leitgeb, E.; Sheikh Muhammad, S.; Kandu, G. Weather effects on hybrid FSO/RF communication link. IEEE J. Sel. Areas Commun. 2009, 27, 1687-1697. [CrossRef]

3. Khalid, H.; Waris, F.; Asif, H.M. Design of an Integrated Power Line Communication (PLC)-Visible Light Communication (VLC) System for Data Communication. Old City Publ. 2018, 40, 107-125.

4. Amirabadi, M.A.; Vakili, V.T. A novel hybrid FSO/RF communication system with receive diversity. IEEE Photonics Technol. Lett. 2018. [CrossRef]

5. Kim, I.I.; Korevaar, E. Availability of free-space optics (FSO) and hybrid FSO/RF systems. In Proceedings of the International Society for Optics and Photonics, Optical Wireless Communications IV, Denver, CO, USA, 27 November 2001; Volume 4530.

6. Rakia, T.; Yang, H.C.; Alouini, M.S.; Gebali, F. Outage analysisof practical FSO/RF hybrid system with adaptive combining. IEEE Commun. Lett. 2015, 19, 1366-1369. [CrossRef]

7. Wei, C.; Zhang, Z. Analysis of Dual-Hop AF Relay Systems in Mixed RF and FSO Links. arXiv 2017, arXiv:1711.09520v1.

8. Amirabadi, M.A. Performance Analysis of a Novel Hybrid FSO/RF Communication System. Available online: https://arxiv.org/ftp/arxiv/papers/1802/1802.07160.pdf (accessed on 18 February 2018). 
9. Bag, B.; Das, A.; Ansari, I.S.; Prokes, A.; Bose, C.; Chandra, A. Performance Analysis of Hybrid FSO Systems Using FSO/RF-FSO Link Adaptation. IEEE Photonics J. 2018, 10, 1-17. [CrossRef]

10. Shakir, W.M.R. Performance evaluation of a selective combining scheme for the hybrid FSO/RF system. IEEE Photonics J. 2018, 10, 1-10. [CrossRef]

11. Abdul Hussein, A.; Oka, A.; Nguyen, T.T.; Lampe, L. Rateless coding for hybrid free-space optical and radio-frequency communication. IEEE Trans.Wirel.Commun. 2010, 9, 907-913. [CrossRef]

12. Zhang, W.; Hranilovic, S.; Shi, C. Soft-switching hybrid FSO/RF links using short-length raptor codes: Design and implementation. IEEE J. Sel. Areas Commun. 2019, 27, 1698-1708. [CrossRef]

13. Nistazakis, H.E.; Tombras, G.S.; Tsigopoulos, A.D.; Karagianni, E.A.; Fafalios, M.E. Capacity estimation of optical wireless communication systems over moderate to strong turbulence channels. J. Commun. Netw. 2019, 11, 387-392. [CrossRef]

14. Al-Habash, M.A.; Andrews, L.C.; Phillips, R.L. Mathematical model for the irradiance probability density function of a laser beam propagating through turbulent media. Opt. Eng. 2001, 40, 1554-1562. [CrossRef]

15. Nistazakis, H.E.; Stassinakis, A.N.; Muhammad, S.S.; Tombras, G.S. BER estimation for multi hop RoFSO QAM or PSK OFDM communication systems over gamma gammaor exponentially modeled turbulence channels. Elsevier Opt. Laser Technol. 2014, 64, 106-112. [CrossRef]

16. Gappmair, W.; Nistazakis, H.E. Subcarrier PSK performance in terrestrial FSO links impaired by gamma-gamma fading, pointing errors and phase noise. IEEE/OSA J. Lightwave Technol. 2017, 35, 1624-1632. [CrossRef]

17. Manning, R. An anisotropic turbulence model for wave propagation near the surface of earth. IEEE Trans. Antennas Propag. 1986, 34, 258-261. [CrossRef]

18. Wu, C.; Paulson, D.A.; van Iersel, M.; Coffaro, J.; Beason, M.; Smith, C.; Crabbs, R.F.; Phillips, R.; Andrews, L.; Davis, C.C. Near ground surface turbulence measurements and validation: A comparison between different systems. In Proceedings of the SPIE, Laser Communication and Propagation through the Atmosphere and Oceans VII, San Diego, CA, USA, 21-23 August 2018; Volume 10770. [CrossRef]

19. Usman, M.; Yang, H.-C.; Alouini, M.-S. Practical Switching-Based Hybrid FSO/RF Transmission and Its Performance Analysis. IEEE Photonics J. 2014, 6, 1-13. [CrossRef]

20. Nistazakis, H.E.; Tsiftsis, T.A.; Tombras, G.S. Performance analysis of free space optical communication systems over atmospheric turbulence channels. IET Commun. 2009, 3, 1402-1409. [CrossRef]

21. Adamchik, V.S.; Marichev, O.I. The algorithm for calculating integrals of hypergeometric type function and its realization in reduce system. In Proceedings of the International Symposium on Symbolic and Algebraic Computation, Tokyo, Japan, 20-24 August 1990; ACM: New York, NY, USA; pp. 212-224.

22. Papoulis, A.; Pillai, S.U. Probability, Random Variables and Stochastic Processes; McGraw-Hill Europe: New York, NY, USA, 2002; ISBN 978-0071226615.

23. Elganimi, T.Y. Performance comparison between OOK, PPM and PAM modulation schemes for free space optical (FSO) communication systems: Analytical study. Int. J. Comput. Appl. 2013, 79, 22-27. 